\title{
Icariin possesses chondroprotective efficacy in a rat model of dexamethasone-induced cartilage injury through the activation of miR-206 targeting of cathepsin $\mathrm{K}$
}

\author{
NING LIU* ${ }^{*}$ TAO ZHANG ${ }^{*}$, BO-RAN CAO, FEI-YU LUAN, RUI-XUAN LIU, \\ HAO-RONG YIN and WEN-BO WANG \\ Department of Orthopedic Surgery, The First Affiliated Hospital of \\ Harbin Medical University, Harbin, Heilongjiang 150001, P.R. China
}

Received March 23, 2017; Accepted October 17, 2017

DOI: $10.3892 /$ ijmm.2017.3289

\begin{abstract}
The present study aimed to investigate the articular cartilage and chondrocytes of dexamethasone (DXM)-induced cartilage injuries in rats in response to treatment with icariin, as well as the implicated molecular mechanism. Effects of icariin on bone metabolism and articular cartilage in experimental rats were investigated. Subsequently, the present study assessed dysregulated microRNA (miRNA) in the articular cartilage of experimental rats, and validated the significant miRNA and their targets. Finally, the effects of icariin on chondrocytes in experimental rats and the implicated molecular mechanism were explored. Quantitative polymerase chain reaction demonstrated that icariin significantly reversed DXM-induced bone degradation and stimulated bone regeneration. In addition, some notable changes in articular cartilage were also observed following continuous administration of icariin to DXM-treated rats, including enhanced cartilage area (revealed by safranin-O staining), substantial decrements in serum concentrations of deoxypyridinoline and C-terminal telopeptide of type II collagen, reduced expression of collagen type I and matrix metalloproteinase-13, as well as elevated expression of transforming growth factor- $\beta$. Furthermore, miR-206 was determined to be significantly upregulated in the icariin group compared with the DXM-treated group. A luciferase assay further validated cathepsin $\mathrm{K}$ as the target RNA of miR-206. Additionally, icariin (100 $\mu \mathrm{M})$ facilitated the viability of chondrocytes and reduced apoptotic chondrocytes. More importantly, icariin $(100 \mu \mathrm{M})$ not only abolished
\end{abstract}

Correspondence to: Dr Wen-Bo Wang, Department of Orthopedic Surgery, The First Affiliated Hospital of Harbin Medical University, 23 Youzheng Road, Harbin, Heilongjiang 150001, P.R. China

E-mail:wenbo_wang80@sohu.com

${ }^{*}$ Contributed equally

Key words: icariin, articular cartilage, chondrocytes, miR-206, cathepsin $\mathrm{K}$ the inhibition effect of DXM on miR-206 expression in chondrocytes, but also eliminated the enhancing effect of DXM on cathepsin K expression. Overall, the present study identified icariin as a novel therapeutic agent in DXM-induced cartilage injury in rats, and revealed that the activation of miR-206 targeting of cathepsin $\mathrm{K}$ may be responsible for the chondroprotective efficacy of icariin.

\section{Introduction}

Cartilages are avascular, tough, flexible, fibrous connective tissues that have multiple roles. Transient cartilages are a major component of embryonic skeletons and offer a model for bone formation, while permanent cartilages exhibit various biomechanical characteristics, including elastic cartilage, hyaline cartilage and fibrocartilage (1). The present study focused on articular cartilage, a sort of hyaline cartilage, which serves crucial roles in supporting and allotting forces that emerge during joint loading, and supplying a frictionless lubricating surface to protect the joint from wear or degradation (2). On account of the deficiency of nerves, blood vessels, and the inherently poor differentiating ability of chondrocytes, damaged articular cartilage has an extremely limited capacity to self-heal $(3,4)$. Finally, these cartilage injuries result in premature joint degeneration and posttraumatic arthritis (5). Despite the development of numerous methods for the restoration of cartilage lesions, some shortcomings remain (6). Therefore, the search for novel therapies with low cost, stable activity and security for cartilage injuries is required.

Herb Epimedium (HEP), a traditional Chinese medicine, has been widely used for arthritis treatment in China, Korea and Japan $(7,8)$. Icariin is the major effective constituent of HEP $(9,10)$. Research has indicated that icariin promoted osteoblast differentiation through inducing BMP-2, BMP-4 and SMAD4 expression (11). Additionally, in bone tissue engineering, a study by Zhao et al (12) demonstrated efficient osteoinductivity of icariin that was able to enhance in vivo bone formation. In a murine model of dexamethasone (DXM)-induced osteoporosis, icariin exerted protective effects against bone deteriorations and promoted bone remodeling, with significant decreases in bone resorption markers 
C-terminal telopeptide of type II collagen (CTX-II) and tartrate-resistant acid phosphatase (TRAP)-5b being observed (13). Icariin has been demonstrated to be a safe and powerful chondrocyte anabolic agent to stimulate chondrocyte proliferation and attenuate extracellular matrix (ECM) degradation $(14,15)$. ECM, in response to the properties of cartilage, is synthesized by chondrocytes (16), thus icariin may be a potential catalyst for chondrogenesis in cartilage tissue engineering. In the present study, the effects of icariin were assessed in a rat model of DXM-induced cartilage injury.

\section{Materials and methods}

Animal experiments and drug administration. A total of 90 6-week-old male Wistar rats, weighing 160-230 g, were obtained from the Centre of Laboratory Animals of Harbin Medical University (Harbin, China). The rats were given free access to food and water and were caged individually in a controlled temperature $\left(21-22^{\circ} \mathrm{C}\right)$ in $50-60 \%$ humidity, with an artificial light cycle (12-h light/dark). All rats were acclimated for 5 days and randomized into the following three groups ( $n=10 /$ group): vehicle (control), DXM, and icariin (DXM group treated with icariin). The vehicle group received normal feed for 12 weeks; the DXM group were injected intramuscularly with $5 \mathrm{mg} / \mathrm{kg}$ body weight DXM (Sigma-Aldrich; Merck KGaA, Darmstadt, Germany) three times a week for 12 weeks; rats in the icariin group were dosed orally for 12 weeks with icariin (100 mg/kg/day; Sigma-Aldrich; Merck KGaA) combined with DXM for 12 weeks. The animal protocol was approved by the Committee on the Ethics of Animal Experiments of Harbin Medical University.

Cell culture. Articular chondrocytes were isolated from the knee joints of rats in the vehicle group as previously described (17), with some alterations. Articular cartilage tissues were cut into small pieces $\left(<1 \mathrm{~mm}^{3}\right)$ and digested with $0.2 \%$ trypsin and $0.2 \%$ type II collagenase for $30 \mathrm{~min}$ and $2 \mathrm{~h}$, respectively. The released cells were maintained in Dulbecco's modified Eagle's medium/F12 supplemented with $10 \%$ fetal bovine serum (FBS; Sigma-Aldrich; Merck KGaA), 100 U/ml penicillin and $100 \mu \mathrm{g} / \mathrm{ml}$ streptomycin at $37^{\circ} \mathrm{C}$ in a humidified atmosphere of $\mathrm{CO}_{2}$ in air. When the cells reached $\sim 90 \%$ confluence, they were treated with various concentrations of $\operatorname{DXM}(0,10,50$ and $100 \mu \mathrm{M})$ for $48 \mathrm{~h}$ at $37^{\circ} \mathrm{C}$. For miR-206 inhibitor treatment, cells were transfected with anti-miR-206 inhibitor (200 nM; Riboxx GmbH, Radebeul, Germany) using GeneCellin transfection reagent (BioCellChallenge, Toulon, France), according to manufacturer's instructions. A total of $48 \mathrm{~h}$ after transfection, cells were harvested and used for further experiments. The sequence of the miR-206 inhibitor was 5'-CCACACACUUCCUUACAUUCCA-3'.

$R N A$ isolation and reverse transcription-quantitative polymerase chain reaction ( $R T-q P C R)$. RT-qPCR was performed as previously reported (18), with some changes. Briefly, the cartilage tissues were crushed under liquid nitrogen conditions and total RNA extraction was performed with TRIzol reagent (Invitrogen; Thermo Fisher Scientific, Inc., Waltham, MA, USA), according to manufacturer's instructions. Samples (1 $\mu \mathrm{g}$ RNA) were reverse-transcribed using a Reverse Transcription
System for real-time PCR (Takara Biotechnology Co., Ltd., Dalian, China), according to the manufacturer's instructions. Synthesized cDNA was used in qPCR experiments using SsoFast $^{\mathrm{TM}}$ EvaGreen Supermix (Bio-Rad Laboratories, Inc., Hercules, CA, USA). qPCR was performed with the following primers: alkaline phosphatase (ALP) sense, 5'-GCCCTCTCCA AGACATATA-3' and antisense, 5'-CCATGATCACGTCG ATATCC-3'; TRAP sense, 5'-AGATCTCCAAGCGCTGG AAC-3' and antisense, 5'-AGGTAGCCGTTGGGGACCTT-3'; osteocalcin sense, 5'-ATGAGAGCCCTCACACTCCTC-3' and antisense, 5'-CTAGACCGGGCCGTAGAAGCG-3'; cathepsin $\mathrm{K}$ sense, 5'-GGGAGACATGACCAGCGAAG-3' and antisense, 5'-CTGAAAGCCCAACAGGAACC-3'; collagen type I (Col-1) sense, 5'-TCCTGCCGATGTCGCTATC-3' and antisense, 5'-CCATGTAGGCTACGCTGTTCTTG-3'; matrix metalloproteinase (MMP)-13 sense, 5'-CCCTGGAGCCCTG ATGTTT-3' and antisense, 5'-CTCTGGTGTTTTGGGGT GCT-3'; transforming growth factor (TGF)- $\beta 1$ sense, 5 '-CCAAG GAGACGGAATACAGG-3' and antisense, 5'-GTGTTGGTTG TAGAGGGCAAG-3'; miR-612 sense, 5'-CAGGGCTTCTGAG CTCCTTA-3' and antisense, 5'-TGAGAGTCCTGTCCTG GCTG-3'; miR-206 sense, 5'-GATTCGCCAAAGGAAAT AGC-3' and antisense, 5'-GTTACAAGGTCATCCAAGAC-3'; miR-28-5p sense, 5'-GTGCACTGTCACGGGTTTTC-3' and antisense, 5'-CCTCTGCAGCCTGGTGAC-3'; miR-714 sense, 5'-CTGCAAGGGTGGAGGTGTAG-3' and antisense, 5'-AGG CAGTGGTCTAAACTCGC-3'; miR-7a sense, 5'-TGTTGGCC TAGTTCTGTGTGG-3' and antisense, 5'-GGCAGACTGTG ATTTGTTGTCG-3'; miR-365 sense, 5'-AAATGAGGGACT TTCAGGGGC-3' and antisense, 5'-AACAATAAGGATTTTT AGGGGCATT-3'; and GAPDH sense, 5'-GTCGGTGTGAAC GGATTTG-3' and antisense, 5'-CTTGCGTGGGTAGAGT CAT-3'. qPCR amplification was performed with an initial denaturation at $95^{\circ} \mathrm{C}$ for $5 \mathrm{~min}$, followed by 27 cycles of $95^{\circ} \mathrm{C}$ for $1 \mathrm{~min}, 65^{\circ} \mathrm{C}$ for $1 \mathrm{~min}$ and $72^{\circ} \mathrm{C}$ for $1 \mathrm{~min}$, with a final extension step of $72^{\circ} \mathrm{C}$ for $10 \mathrm{~min}$. Results were analyzed with Opticon Monitor software 3.1 (Bio-Rad Laboratories, Inc.). Specificity was determined by $1 \%$ agarose gel electrophoretic analysis of the reaction products. GAPDH was used as an internal standard. Data were analyzed using the $2^{-\Delta \Delta \mathrm{Cq}}$ method, as previously described (19).

Western blotting. Western blotting was performed as previously reported (20). Briefly, proteins were extracted by lysing cells in buffer (50 mM Tris pH 7.4, $150 \mathrm{mM} \mathrm{NaCl,} \mathrm{0.5 \%} \mathrm{NP-40,}$ $50 \mathrm{mM} \mathrm{NaF}, 1 \mathrm{mM} \mathrm{Na} \mathrm{VO}_{4}, 1 \mathrm{mM}$ phenylmethylsulfonyl fluoride, $25 \mathrm{mg} / \mathrm{ml}$ leupeptin and $25 \mathrm{mg} / \mathrm{ml}$ aprotinin). Protein concentration was determined using a bicinchoninic acid protein assay (Bio-Rad Laboratories, Inc.). Subsequently, proteins $(20 \mu \mathrm{g})$ were separated by $10 \%$ SDS-PAGE mini-gel and transferred to a polyvinylidene difluoride membrane (EMD Millipore, Billerica, MA, USA) for $60 \mathrm{~min}$ at $100 \mathrm{~V}$. Following incubation in blocking buffer (Tris-buffered saline containing $150 \mathrm{mM} \mathrm{NaCl}, 50 \mathrm{nM}$ Tris and $0.05 \%$ Tween-20; $\mathrm{pH} 7.5$ ) for $1 \mathrm{~h}$ at room temperature, the membrane was hybridized in blocking buffer with specific primary antibodies against cathepsin K (sc-48353; 1:100), B-cell lymphoma 2 (Bcl-2; sc-56015; 1:50), Bcl-2-associated X protein (Bax; sc-70407; 1:200), caspase-3 (sc-271759; 1:200), caspase-9 (sc-8355; 1:200) and $\beta$-actin (sc-70319; $1: 200)$ overnight at $4^{\circ} \mathrm{C}$. 
Subsequently, the membrane was incubated with secondary antibodies labeled with horseradish peroxidase (sc-516102; 1:200) for $1 \mathrm{~h}$ at room temperature, followed by detection with an enhanced chemiluminescence system (GE Healthcare, Chicago, IL, USA). All antibodies were obtained from Santa Cruz Biotechnology, Inc. (Dallas, TX, USA). $\beta$-actin was used as the protein loading control. Band intensities were quantified by immunoblot densitometry using Image-Pro Plus 4.5 (Media Cybernetics, Inc., Rockville, MD, USA).

Serum biochemical markers of cartilage metabolism. Anesthetized rats were placed in a euthanasia chamber. Directly after aspiration, blood was transferred to plain tubes. Serum was centrifuged at $2,000 \times \mathrm{g}$ for $1 \mathrm{~min}$ at $4^{\circ} \mathrm{C}$, and stored at $-80^{\circ} \mathrm{C}$ until analysis. Serum levels of CTX-II and deoxypyridinoline (DPD) were measured using a rat ELISA assay (60-2700; Immutopics, Inc., San Clemente, CA, USA), as previously described (21).

Histomorphological analysis. Cartilage tissues were dissected along the axial plane into pieces of $10 \times 5 \times 7 \mathrm{~mm}$ with a thin layer of subchondral bone and were fixed in $4 \%$ formaldehyde for over $24 \mathrm{~h}$ at room temperature. Following decalcification in $10 \%$ EDTA solution for over 2 weeks, the samples were embedded in paraffin. The specimens were cut into $4-\mu \mathrm{m}$ sections and stained with safranin- $\mathrm{O}$ for $5 \mathrm{~min}$ at room temperature. To evaluate the volume of cartilage formation, the area occupied by chondrocytes and cartilage matrix stained with safranin-O was quantified using an image analysis system (Image J version $1.43 \mathrm{u}$; National Institutes of Health, Bethesda, MD, USA).

Luciferase activity assay. The potential binding sites between cathepsin K and miR-206 were predicted using TargetScan (targetscan.org/mamm_31/). A luciferase assay was used to validate cathepsin $\mathrm{K}$ as a target of miR-206. Articular chondrocytes $\left(\sim 7.5 \times 10^{4}\right.$ cells/well) were seeded in 12 -well plates, then transfected with the wild-type, mutant cathepsin K 3 ' untranslated region (3'UTR) or the vector alone (co-transfected using pGL3-control) using Lipofectamine 2000 (Invitrogen; Thermo Fisher Scientific, Inc.), in accordance with the manufacturer's instructions. After $48 \mathrm{~h}$, cells were lysed, then the firefly and Renilla luciferase activities were measured using the dual luciferase reporter assay kit (Promega, Madison, WI, USA) on a luminometer (Orion II Microplate Luminometer; Berthold Detection Systems GmbH, Pforzheim, Germany). To check the specificity of the miR-206 effect, chondrocytes were co-transfected with wild-type cathepsin K 3'UTR or miR-206 before luciferase activities were measured. Control cells were transfected with the scramble sequence. Renilla reporter luciferase activity was normalized to the firefly luciferase activity.

Cell viability and apoptosis assays. Cell viability was assessed by LIVE/DEAD assay as previously reported (22). Briefly, cells were stained using a LIVE/DEAD stain kit (Thermo Fisher Scientific, Inc.), according to the manufacturer's instructions. This kit contains two fluorescent dyes, calcein-AM to stain living cells green and ethidium homodimer-1 (Ethd-1) to stain dead cells red. Following staining, samples were observed through an epifluorescent microscope (Carl Zeiss AG,
Oberkochen, Germany) at a magnification of x200. For each well, at least five different fields were examined, and a minimum of 1,000 cells were counted to determine the fraction of calcein-AM-positive cells vs. Ethd-1-positive cells.

In addition, to evaluate apoptotic activity of chondrocytes, terminal deoxynucleotidyl-transferase-mediated dUTP nick end labelling (TUNEL) staining was performed, as previously described (23). Frozen cartilage sections were fixed with $4 \%$ methanol-free formaldehyde solution in PBS for $10 \mathrm{~min}$ at room temperature, then washed with PBS three times. The DNA fragments were labeled with fluorescein-12-dUTP in terminal deoxynucleotidyl transferase incubation buffer (Promega) in a humidified chamber $\left(37^{\circ} \mathrm{C}\right.$ for $\left.60 \mathrm{~min}\right)$ to avoid exposure to light. The reactions were stopped by transferring the slides to $\mathrm{SSC}$ buffer $(0.3 \mathrm{M} \mathrm{NaCl}, 0.03 \mathrm{M}$ sodium citrate, $\mathrm{pH}$ 7.0) for $15 \mathrm{~min}$ and washing with PBS to remove unincorporated fluorescein-12-dUTP. The slides were then counterstained with $1 \mu \mathrm{g} / \mathrm{ml}$ 4',6-diamidino-2-phenylindole (Vector Laboratories, Inc., Burlingame, CA, USA) for $5 \mathrm{~min}$ at room temperature to provide a blue background. The green fluorescence of apoptotic cells (fluorescein-12-dUTP) may be detected using a fluorescence microscope at $520 \mathrm{~nm}$.

Caspase-3 activity. Caspase-3 activity was evaluated as previously reported (24). Assays were performed in 96-well microtiter plates by incubating $20 \mu \mathrm{g}$ cell lysate in $100 \mu \mathrm{l}$ reaction buffer (1\% NP-40, $20 \mathrm{mM}$ Tris- $\mathrm{HCl} \mathrm{pH}$ 7.5, $137 \mathrm{mM}$ $\mathrm{NaCl}$ and $10 \%$ glycerol) containing $5 \mu \mathrm{M}$ caspase-3 substrate (Ac-DEVD-pNA; Sigma-Aldrich; Merck KGaA). Lysates were incubated at $37^{\circ} \mathrm{C}$ for $2 \mathrm{~h}$. Subsequently, the absorbance was measured at $405 \mathrm{~nm}$ with a spectrophotometer.

Statistical analysis. All experiments were conducted at least three times. Data were presented as the mean \pm standard error of the mean. Statistical analyses were performed using PRISM version 4.0 (GraphPad Software, Inc., La Jolla, CA, USA). The differences among groups were analyzed by one-way analysis of variance, followed by Tukey's multiple comparison test. $\mathrm{P}<0.05$ was considered to indicate a statistically significant difference.

\section{Results}

Effects of icariin on biomarkers of bone metabolism in experimental rats. The expression of biochemical markers of bone turnover in the three experimental groups, including TRAP as a bone resorption marker, and ALP and osteocalcin as bone formation markers, were first determined using RT-qPCR. Compared with the vehicle control group, the DXM group demonstrated significantly increased expression of ALP and TRAP; however, icariin treatment significantly eliminated the enhancing effect of DXM on the expression of ALP and TRAP compared with the DXM group (Fig. 1A and B). In contrast, icariin significantly stimulated osteocalcin expression following DXM treatment compared with the levels observed in the DXM treatment group (Fig. 1C). Additionally, it was also determined that icariin abolished the promotion effect of DXM on cathepsin K expression, an enzyme expressed by osteoclasts in response to bone resorption, at the mRNA and protein expression levels (Fig. 1D and E). 


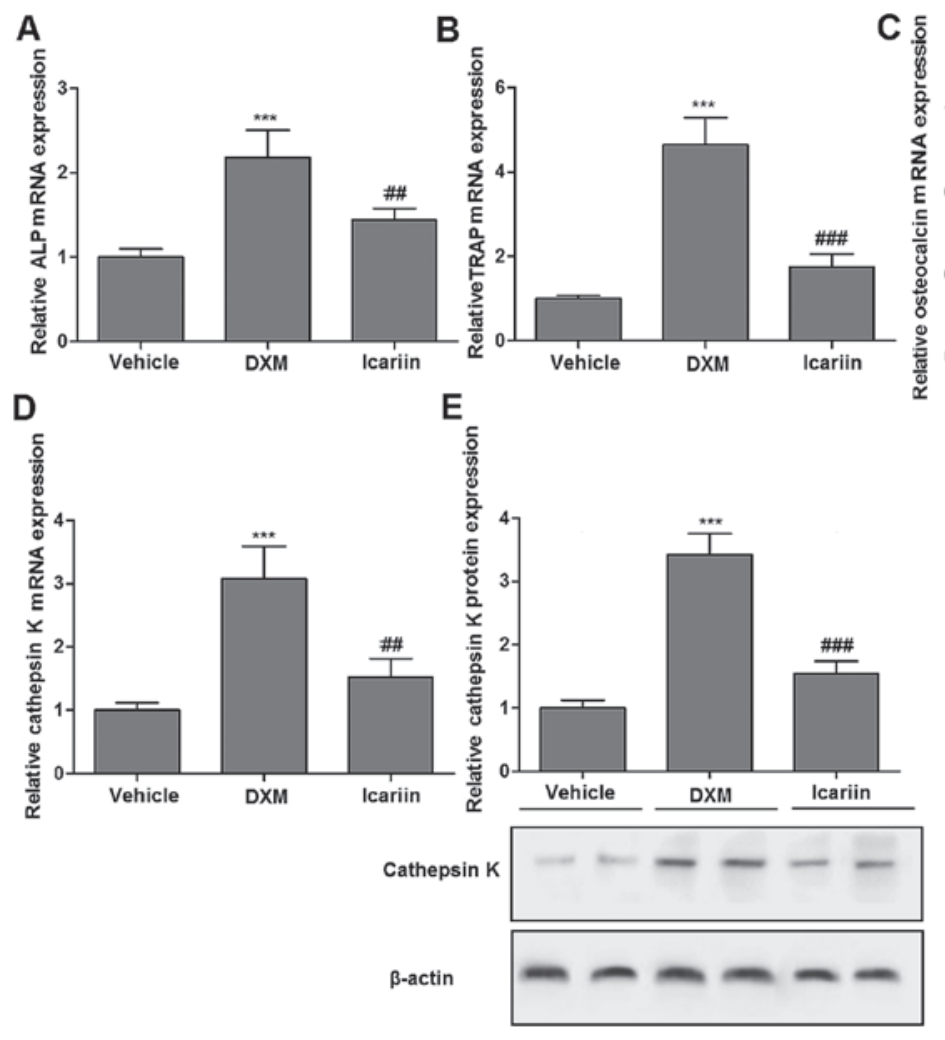

Figure 1. mRNA expression of (A) ALP, (B) TRAP, (C) osteocalcin and (D) cathepsin K in the three experimental groups measured by reverse transcription-quantitative polymerase chain reaction. Compared with the vehicle control group, the DXM group demonstrated significantly increased expression levels of ALP, TRAP and cathepsin K, and decreased osteocalcin expression. However, icariin treatment significantly eliminated the enhancing effect of DXM on the expression of ALP, TRAP and cathepsin K, and stimulated osteocalcin expression. (E) Protein expression of cathepsin K in the three experimental groups measured by western blotting. Icariin significantly abolished the promotion effect of DXM on cathepsin $\mathrm{K}$. Values are expressed as the mean \pm standard error of the mean ( $\mathrm{n}=10$ /group). ${ }^{* * * *} \mathrm{P}<0.001$ vs. vehicle group; ${ }^{\# \#} \mathrm{P}<0.01$ and ${ }^{\# \# \#} \mathrm{P}<0.001$ vs. DXM group. ALP, alkaline phosphatase; TRAP, tartrate-resistant acid phosphatase; DXM, dexamethasone.

Effects of icariin on cartilage metabolism in experimental rats. The present study assessed the effects of icariin on articular cartilage in the three groups. As demonstrated in Fig. 2A, a small volume of cartilage formation was observed in the DXM group by safranin-O staining, relative to the control group; however, continuous administration of icariin enhanced the volume of cartilage formation. Similarly, administration of icariin to DXM-treated rats significantly elevated the reduced cartilage area (occupied by chondrocytes and cartilage matrix dyed with safranin-O; Fig. 2B). More importantly, icariin significantly reversed the DXM-induced increase in serum concentrations of DPD and CTX-II, the commonly used serum markers of cartilage metabolism, in experimental rats (Fig. 2C and D). Furthermore, the expression of Col-1, MMP-13 and TGF- $\beta$ in the three experimental groups, which are cartilage metabolism-related genes, were determined. Icariin significantly reduced the elevated expression of Col-1 and MMP-13 induced by DXM, but promoted TGF- $\beta$ expression (Fig. $2 \mathrm{E}-\mathrm{G}$ ).

Validation of dysregulated microRNA (miRNA) in articular cartilage of experimental rats. The results in Fig. 2 demonstrated the beneficial effect of icariin on cartilage metabolism in DXM-treated rats, thus it was speculated whether icariin affected articular cartilage of experimental rats at the miRNA level. The relative expression of the miRNA in articular cartilage of all experimental rats were presented as a heatmap (Fig. 3A). The deeper the color, the higher the expression. A total of five miRNA were further determined to be significantly upregulated in the icariin group, compared with the reduced levels in the DXM-treated group, including miR-612, -206, -28-5p, -7a and -365 (Fig. 3B-G). In view of the role of miR-206 as a key regulator during osteoblast differentiation (25), miR-206 was selected as the candidate for the following experiments.

miR-206 targets cathepsin $K$. To determine whether miR-206 regulated cathepsin $\mathrm{K}$ through the predicted binding sites in its 3'UTR (Fig. 4A), two luciferase constructs were employed by incorporating wild-type or mutant 3'UTR of cathepsin K, which expressed luciferase unless repressed by the incorporated 3'UTR. As demonstrated in Fig. 4B, co-transfection with the pMIR-REPORT construct containing mutant cathepsin $\mathrm{K}$ 3'UTR and PLemiR-206 did not exhibit a significant difference compared with the control; however, co-transfection with the luciferase construct containing wild-type cathepsin K 3'UTR and PLemiR-206 resulted in an $70 \%$ decline in luciferase activity compared with the control. As demonstrated in Fig. 4C and D, compared with the control group, miR-206 overexpression significantly decreased the expression of cathepsin K; however, the inhibition of miR-206 elevated the level of cathepsin K, both at the mRNA and protein expression levels.

Effects of icariin on chondrocytes in experimental rats and the implicated molecular mechanism. Finally, the present 

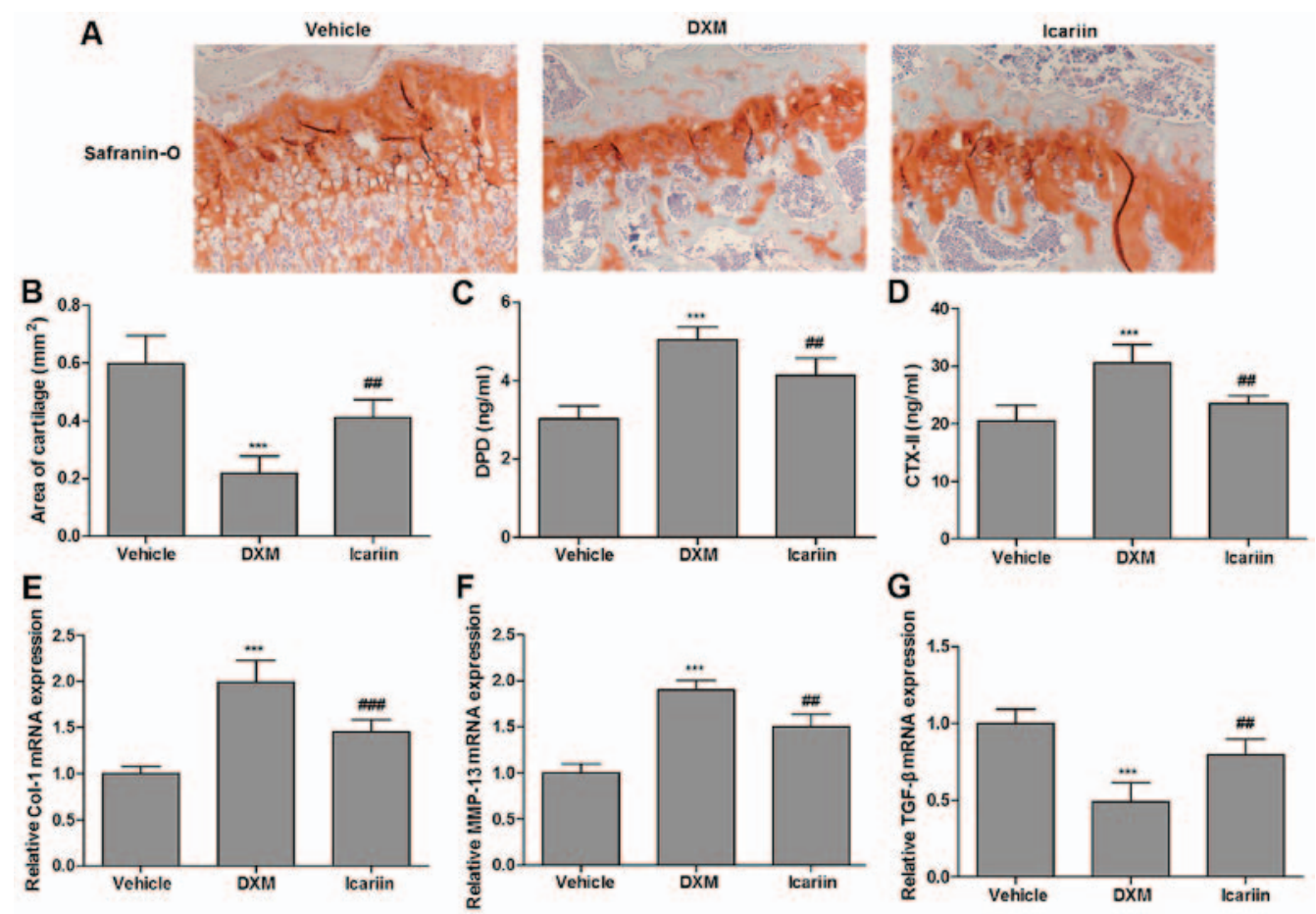

Figure 2. (A) Cartilage formation in the three experimental groups assessed by safranin-O staining. Region stained red is cartilage (magnification, $\mathrm{x} 200$ ). (B) Quantitative analysis of cartilage area. Icariin treatment in DXM-treated rats significantly elevated the reduced cartilage area (occupied by chondrocytes and cartilage matrix dyed with safranin-O). Serum concentrations of (C) DPD and (D) CTX-II in the three experimental groups determined by ELISA. Icariin significantly reversed the DXM-induced increase in serum concentrations of DPD and CTX-II in experimental rats. mRNA expression of (E) Col-1, (F) MMP-13 and (G) TGF- $\beta$ in the three experimental groups measured by reverse transcription-quantitative polymerase chain reaction. Icariin significantly reduced the elevated expression of Col-1 and MMP-13 induced by DXM, and promoted TGF- $\beta$ expression. Values are expressed as the mean \pm standard error of the mean ( $\mathrm{n}=10 /$ group). ${ }^{* * * *} \mathrm{P}<0.001$ vs. vehicle group; ${ }^{\# \#} \mathrm{P}<0.01$ and ${ }^{\# \# \#} \mathrm{P}<0.001$ vs. DXM group. DXM, dexamethasone; DPD, deoxypyridinoline; CTX-II, C-terminal telopeptide of type II collagen; Col-1, collagen type I; MMP-13, matrix metalloproteinase-13; TGF- $\beta$, transforming growth factor- $\beta$.
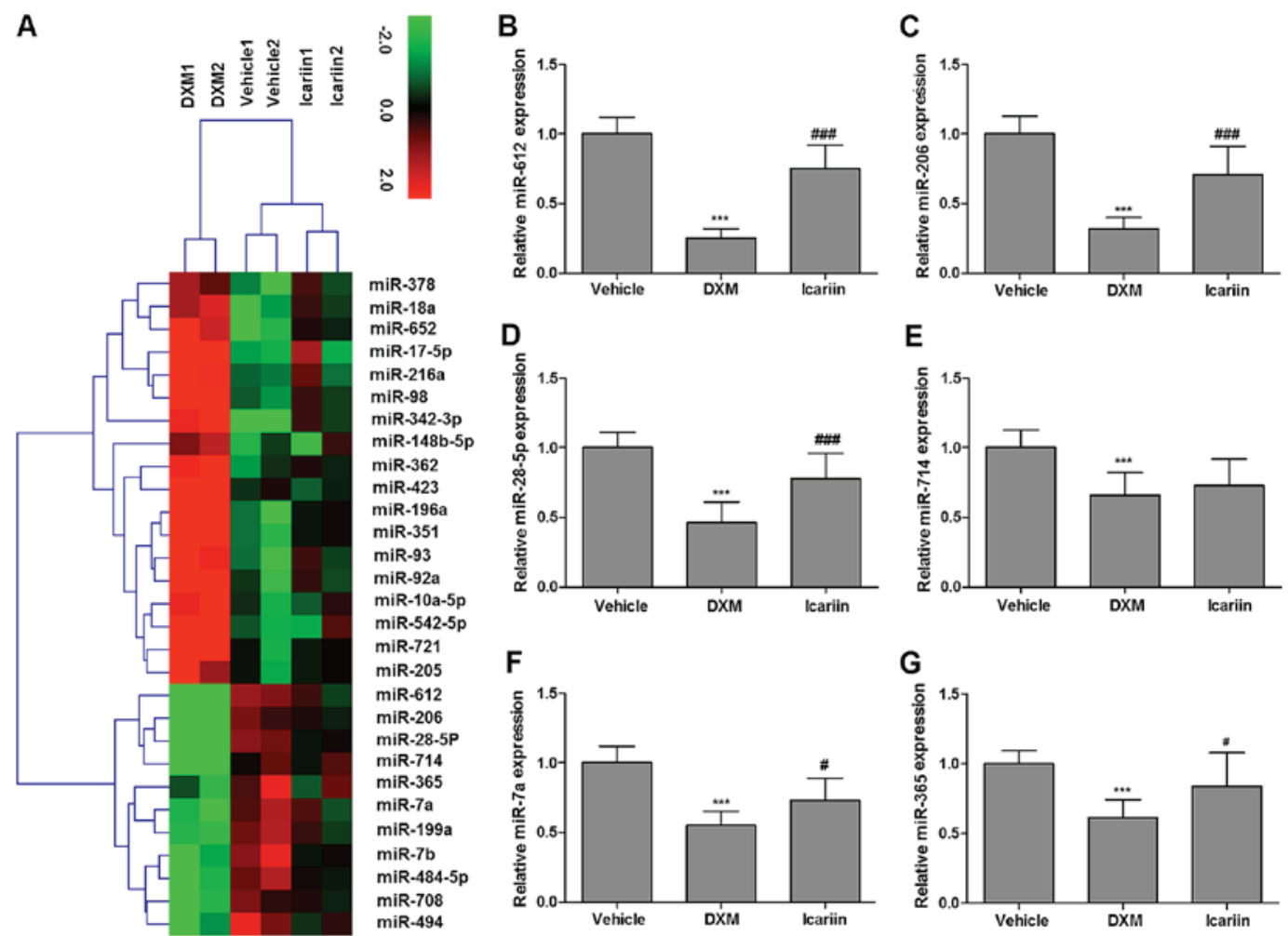

Figure 3. (A) Heatmap of all miR related to articular cartilage in experimental rats. Red indicates high relative expression and green indicates low relative expression. mRNA expression of (B) miR-612, (C) miR-206, (D) miR-28-5p, (E) miR-714, (F) miR-7a and (G) miR-365 in the three experimental groups were determined by reverse transcription-quantitative polymerase chain reaction. DXM significantly reduced miR-612, miR-206, miR-28-5p, miR-7a, miR-714 and miR-365 expression; however, icariin significantly rescued the expression of these miR, except for the expression of miR-714. Values are expressed as the mean \pm standard error of the mean. ${ }^{* * *} \mathrm{P}<0.001$ vs. vehicle group; ${ }^{\#} \mathrm{P}<0.05$ and ${ }^{\# \# \#} \mathrm{P}<0.001$ vs. DXM group. miR, microRNA; DXM, dexamethasone. 
A

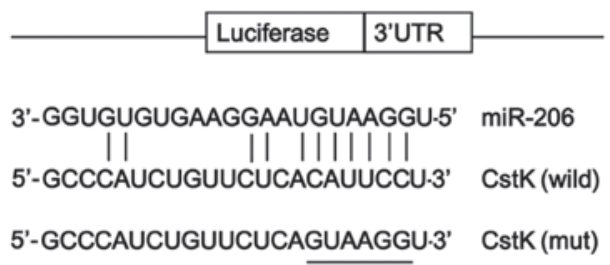

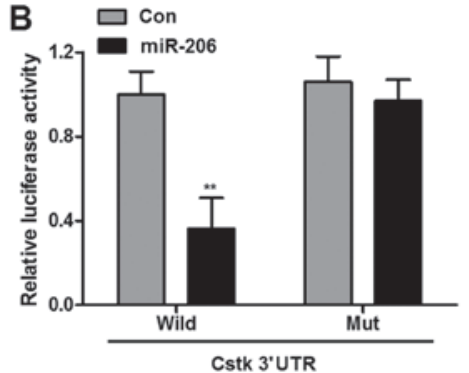

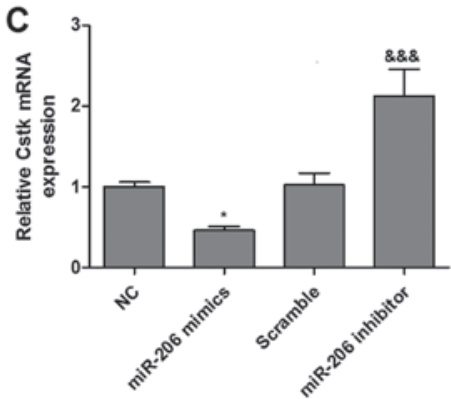

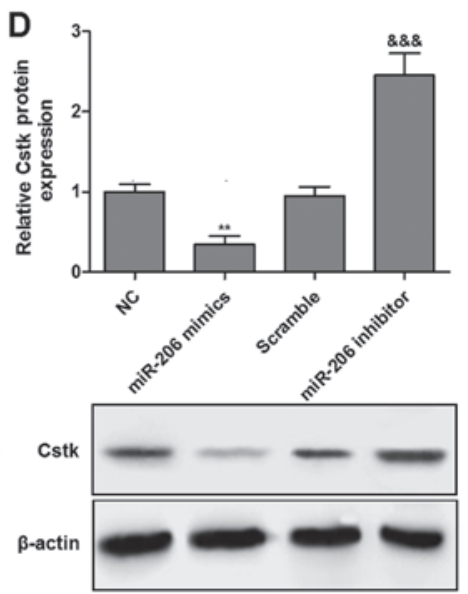

Figure 4. Luciferase activity assay and the expression of Cstk in chondrocytes. (A) Schematic representation of the putative miR-206 binding site in the Cstk 3'UTR in TargetScan. (B) Luciferase activity assay of Cstk. Co-transfection with the luciferase construct containing wild-type Cstk K 3'UTR and PLemiR-206 resulted in a significant decline in luciferase activity compared with the control. miR-206 overexpression significantly decreased the expression of Cstk; however, inhibiting miR-206 elevated the level of Cstk, both at the (C) mRNA and (D) protein levels. mRNA expression of Cstk was measured by reverse transcription-quantitative polymerase chain reaction, and protein expression was measured by western blotting. Values are expressed as the mean \pm standard error of the mean. ${ }^{*} \mathrm{P}<0.05$ and ${ }^{* *} \mathrm{P}<0.01$ vs. con or $\mathrm{NC}$ group; ${ }^{\& \& \&} \mathrm{P}<0.001$ vs. scramble group. Cstk, cathepsin K; UTR, untranslated region; Con, control; NC, normal control; miR, microRNA; wild, wild-type; Mut, mutant.

study assessed the effects of icariin on chondrocytes in the presence of DXM at the indicated concentrations (Fig. 5). As demonstrated in Fig. 5A, the addition of DXM $(100 \mu \mathrm{M})$ to chondrocytes resulted in a maximum reduction in cell viability compared with the control cells; however, this effect was reversed significantly following treatment with icariin $(100 \mu \mathrm{M})$. As demonstrated in Fig. 5B and C, icariin $(100 \mu \mathrm{M})$ significantly suppressed DXM-induced elevation of chondrocyte apoptosis, accompanied by an increase in the level of Bcl-2, and decreases in the levels of Bax, caspase- 3 and caspase-9 (Fig. 5D). RT-qPCR further indicated that icariin $(100 \mu \mathrm{M})$ abolished the maximal inhibition effect of DXM (100 $\mu \mathrm{M})$ on miR-206 expression in chondrocytes (Fig. 5E). Additionally, Fig. 5F and G demonstrated that the dose-dependent addition of DXM significantly elevated the expression of cathepsin $\mathrm{K}$, which peaked at a dose of $100 \mu \mathrm{M}$. This level was significantly decreased following icariin stimulation $(100 \mu \mathrm{M})$.

\section{Discussion}

In bone tissue engineering, the effect of icariin on bone metabolism is of interest, including the stimulation of bone formation and osteoblast differentiation (26), as well as the inhibition of bone resorption and osteoclast differentiation (27). However, little is known about the effect of icariin on cartilage tissue and how icariin acts on cartilage tissue engineering, although icariin has been demonstrated to stimulate chondrocyte proliferation and reduce ECM degradation (15). The present data provided available information about the effect of icariin in rats with cartilage disease, as well as the implicated mechanism. Changes in bone markers not only simply predict the response in bone metabolism (28), but may also be detected earlier than the alterations in bone mineral density (29). The present study first identified that the administration of icariin to DXM-treated rats significantly decreased bone resorption (reduced expression of TRAP and cathepsin K), as well as significantly increased the level of bone formation (elevated expression of osteocalcin), which were consistent with the results observed in mice with DXM-induced osteoporosis (13), suggesting a beneficial effect of icariin on bone generation.

There is increasing evidence supporting the important role of icariin in cartilage tissue engineering. An in vivo study of the effect of icariin on cartilage tissue engineering revealed that icariin stimulated ECM secretion and the expression of cartilage-related genes of chondrocytes, implying the possibility of icariin as a promoter in cartilage tissue engineering (30). A study by Sun et al (31) reported that icariin suppressed bone and cartilage deteriorations in mice with collagen-induced arthritis, suggesting that icariin holds promise as a treatment for patients with joint diseases. A study by Yuan et al (32) reported that integrating icariin into hydrogel scaffolds facilitated the synthesis of cartilage matrix and improved the quality of newly formed cartilage. Furthermore, cytokines, particularly TGF- $\beta$, have been demonstrated to be strong regulators of chondrocyte differentiation and to modulate ECM formation $(33,34)$. A recent 

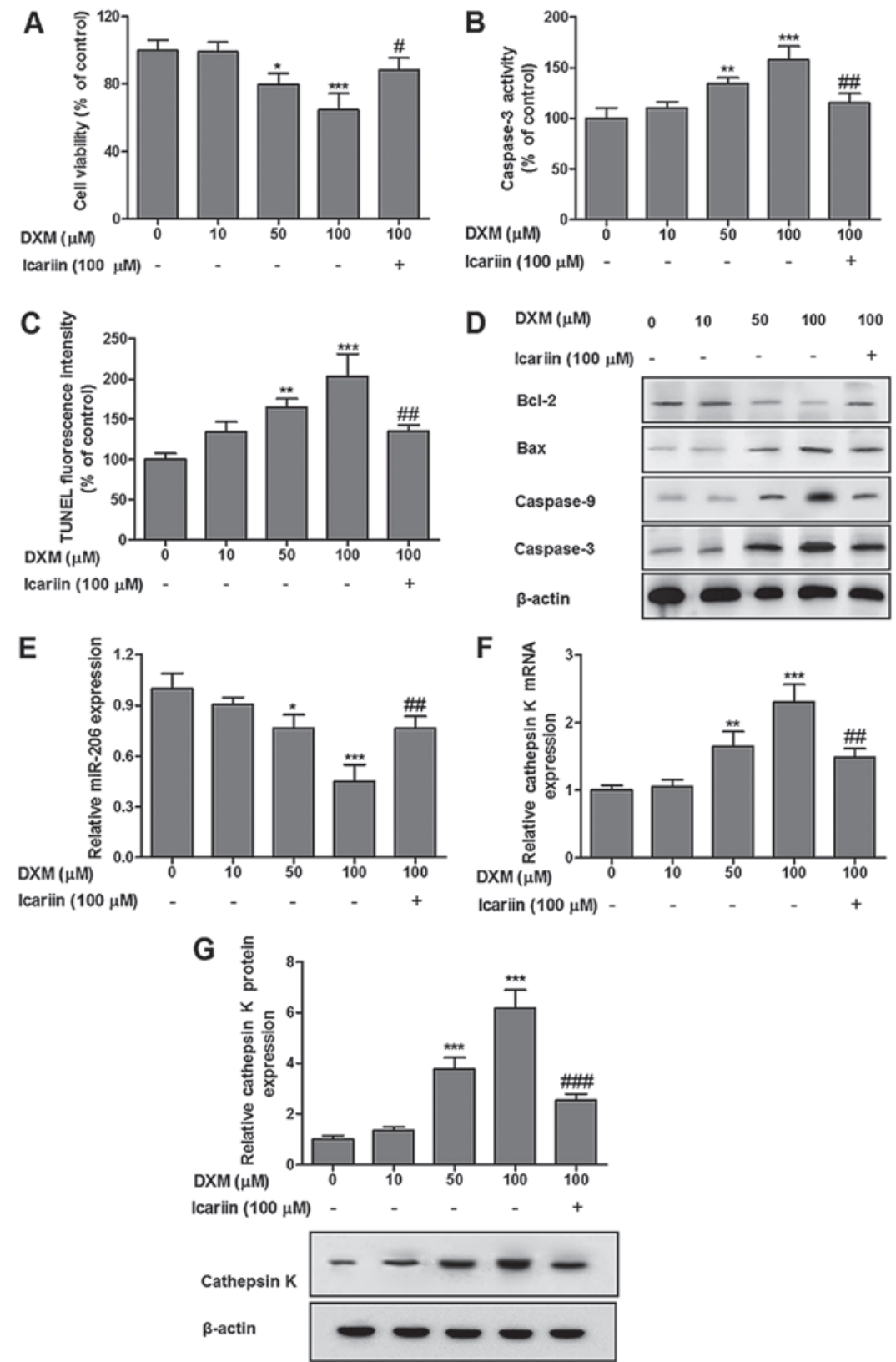

Figure 5. Icariin attenuates DXM-induced dysfunction in rat chondrocytes through the activation of miR-206 targeting to cathepsin K. (A) Chondrocyte viability. Icariin $(100 \mu \mathrm{M})$ significantly reversed DXM-induced reduction of cell viability. (B) Activity of caspase-3. A significant decrease in caspase-3 activity was observed following the addition of icariin $(100 \mu \mathrm{M})$ to DXM-treated chondrocytes. (C) Chondrocyte apoptosis according to TUNEL staining. Icariin $(100 \mu \mathrm{M})$ significantly inhibited the DXM-induced increase in cell apoptosis. (D) Expression levels of proteins implicated in cell apoptosis. (E) mRNA expression of miR-206 measured by reverse transcription-quantitative polymerase chain reaction. Icariin $(100 \mu \mathrm{M})$ abolished the inhibition effect of DXM on miR-206 expression in chondrocytes. Icariin $(100 \mu \mathrm{M})$ significantly lowered the DXM-induced elevated expression of cathepsin K at the (F) mRNA and (G) protein expression levels. Values are expressed as the mean \pm standard error of the mean. ${ }^{*} \mathrm{P}<0.05,{ }^{* *} \mathrm{P}<0.01$ and ${ }^{* * * *} \mathrm{P}<0.001$ vs. DXM $(0 \mu \mathrm{M})$ group; ${ }^{*} \mathrm{P}<0.05$, ${ }^{\# \#} \mathrm{P}<0.01$ and ${ }^{\# \# \#} \mathrm{P}<0.001$ vs. DXM $(100 \mu \mathrm{M})$ group. DXM, dexamethasone; miR, microRNA; TUNEL, terminal deoxynucleotidyl-transferase-mediated dUTP nick end labelling; Bcl-2, B-cell lymphoma 2; Bax, B-cell lymphoma 2-associated X protein.

study has identified that icariin stimulated cartilage repair through the activation of hypoxia inducible factor- $1 \alpha$ in chondrocytes (35). It should be noted that DPD and CTX-II, commonly used serum markers of cartilage metabolism, are important for cartilage turnover. In the present study, as expected, it was observed that icariin markedly enhanced cartilage formation (increased cartilage area) and improved cartilage metabolism (reduced serum concentrations of DPD and CTX-II, reduced expression of Col-1 and MMP-13, and elevated expression of TGF- $\beta$ ) in DXM-treated rats.
More importantly, the present study determined that miR-206 was significantly upregulated following continuous administration of icariin to DXM-treated rats, and cathepsin K was further validated as the target RNA of miR-206. miRNA are small non-coding RNA that act as key post-transcriptional gene regulators and are implicated in various biological processes, including proliferation, development and disease occurrence (36-38). miR-206 has been demonstrated to be a key regulator during osteoblast differentiation (25). Various studies have explored the role of miRNA in cartilage development 
and diseases. A study by Sumiyoshi et al (39) discovered a novel role of miR-181a in cartilage metabolism. A study by Mirzamohammadi et al (40) reported that overexpression of hsa-miR-148a stimulated cartilage formation by inhibiting hypertrophic differentiation and inducing type II collagen production of osteoarthritis chondrocytes. Guérit et al (41) reported that miR-29a was greatly downregulated during chondrogenesis and that overexpression of miR-29a observably inhibited chondrocyte-specific gene expression during chondrogenic differentiation of mesenchymal stem cells. The present study also revealed that the addition of icariin $(100 \mu \mathrm{M})$ to DXM-treated chondrocytes resulted in a significant increase in cell viability, as well as a significant reduction in cell apoptosis, which were extremely similar to the findings in rabbit chondrocytes (42). Additionally, the activation of miR-206 targeting to cathepsin $\mathrm{K}$ following the addition of icariin $(100 \mu \mathrm{M})$ to DXM-treated chondrocytes further suggested a novel miR-206-dependent mechanism that may be responsible for the chondroprotective efficacy of icariin in DXM-induced cartilage injuries in rats.

In conclusion, the present results identified icariin as a potential accelerator exerting protective effects against cartilage degradation and promoting cartilage regeneration in a rat model of DXM-induced cartilage injury. The present study revealed that the activation of miR-206 targeting to cathepsin K, at least partly, was involved in this mechanism.

\section{Acknowledgements}

The present study was supported by the Natural Science Foundation for Young Scholars of Heilongjiang, China (grant no. QC20160117).

\section{References}

1. Dang AC and Kuo AC: Cartilage biomechanics and implications for treatment of cartilage injuries. Oper Tech Orthop 24: 288-292, 2014.

2. Mow VC, Ratcliffe A and Poole AR: Cartilage and diarthrodial joints as paradigms for hierarchical materials and structures. Biomaterials 13: 67-97, 1992.

3. Frenkel SR, Clancy RM, Ricci JL, Di Cesare PE, Rediske JJ and Abramson SB: Effects of nitric oxide on chondrocyte migration, adhesion, and cytoskeletal assembly. Arthritis Rheum 39: 1905-1912, 1996.

4. Klein TJ,Rizzi SC, Reichert JC, Georgi N, Malda J, Schuurman W, Crawford RW and Hutmacher DW: Strategies for zonal cartilage repair using hydrogels. Macromol Biosci 9: 1049-1058, 2009.

5. Furman BD, Strand J, Hembree WC, Ward BD, Guilak F and Olson SA: Joint degeneration following closed intraarticular fracture in the mouse knee: a model of posttraumatic arthritis. J Orthop Res 25: 578-592, 2007.

6. Seyedin MS and Matava M: Cartilage repair methods. US Patent 20070128155 A1. Filed December 7, 2006; issued June 7, 2007. https://www.google.ms/patents/US20070128155.

7. Yu S, Chen K, Li S and Zhang K: In vitro and in vivo studies of the effect of a Chinese herb medicine on osteoclastic bone resorption. Chin J Dent Res 2: 7-11, 1999.

8. Zhang DW, Cheng Y, Wang NL, Zhang JC, Yang MS and Yao XS: Effects of total flavonoids and flavonol glycosides from Epimedium koreanum Nakai on the proliferation and differentiation of primary osteoblasts. Phytomedicine 15: 55-61, 2008.

9. Xie F, Wu CF, Lai WP, Yang XJ, Cheung PY, Yao XS, Leung PC and Wong MS: The osteoprotective effect of Herba epimedii (HEP) extract in vivo and in vitro. Evid Based Complement Alternat Med 2: 353-361, 2005.

10. Qian G, Zhang X, Lu L, Wu X, Li S and Meng J: Regulation of Cbfa1 expression by total flavonoids of Herba epimedii. Endocr J 53: 87-94, 2006.
11. Hsieh TP, Sheu SY, Sun JS, Chen MH and Liu MH: Icariin isolated from Epimedium pubescens regulates osteoblasts anabolism through BMP-2, SMAD4, and Cbfa1 expression. Phytomedicine 17: 414-423, 2010.

12. Zhao J, Ohba S, Komiyama Y, Shinkai M, Chung UI and Nagamune T: Icariin: a potential osteoinductive compound for bone tissue engineering. Tissue Eng Part A 16: 233-243, 2010.

13. Zhang J, Song J and Shao J: Icariin attenuates glucocorticoidinduced bone deteriorations, hypocalcemia and hypercalciuria in mice. Int J Clin Exp Med 8: 7306-7314, 2015.

14. Xu CQ, Liu BJ, Wu JF, Xu YC, Duan XH, Cao YX and Dong JC: Icariin attenuates LPS-induced acute inflammatory responses: involvement of PI3K/Akt and NF-kappaB signaling pathway. Eur J Pharmacol 642: 146-153, 2010.

15. Liu MH, Sun JS, Tsai SW, Sheu SY and Chen MH: Icariin protects murine chondrocytes from lipopolysaccharide-induced inflammatory responses and extracellular matrix degradation. Nutr Res 30: 57-65, 2010.

16. Qi WN and Scully SP: Type II collagen modulates the composition of extracellular matrix synthesized by articular chondrocytes. J Orthop Res 21: 282-289, 2003.

17. Zhou PH, Liu SQ and Peng H: The effect of hyaluronic acid on IL-1 $\beta$-induced chondrocyte apoptosis in a rat model of osteoarthritis. J Orthop Res 26: 1643-1648, 2008.

18. Matsubara J, Yamada Y, Nakajima TE, Kato K, Hamaguchi T, Shirao K, Shimada Y and Shimoda T: Clinical significance of insulin-like growth factor type 1 receptor and epidermal growth factor receptor in patients with advanced gastric cancer. Oncology 74: 76-83, 2008.

19. Livak KJ and Schmittgen TD: Analysis of relative gene expression data using real-time quantitative PCR and the 2(- $\Delta \Delta \mathrm{C}(\mathrm{T}))$ method. Methods 25: 402-408, 2001.

20. Kim JE, Lee MH, Nam DH, Song HK, Kang YS, Lee JE, Kim HW, Cha JJ, Hyun YY, Han SY, et al: Celastrol, an NF- $\mathrm{BB}$ inhibitor, improves insulin resistance and attenuates renal injury in db/db mice. PLoS One 8: e62068-e62068, 2013.

21. DeLaurier A, Jackson B, Pfeiffer D, Ingham K, Horton MA and Price JS: A comparison of methods for measuring serum and urinary markers of bone metabolism in cats. Res Vet Sci 77: 29-39, 2004.

22. Tu Y, Xue H, Francis W, Davies AP, Pallister I, Kanamarlapudi V and Xia Z: Lactoferrin inhibits dexamethasone-induced chondrocyte impairment from osteoarthritic cartilage through up-regulation of extracellular signal-regulated kinase $1 / 2$ and suppression of FASL, FAS, and caspase 3. Biochem Biophys Res Commun 441: 249-255, 2013.

23. Zhou RR,Jia SF, Zhou Z, Wang Y,Bucana CD and Kleinerman ES: Adenovirus-E1A gene therapy enhances the in vivo sensitivity of Ewing's sarcoma to VP-16. Cancer Gene Ther 9: 407-413, 2002.

24. Park JW, Choi YJ, Suh SI, Baek WK, Suh MH, Jin IN, Min DS Woo JH, Chang JS, Passaniti A, et al: Bcl-2 overexpression attenuates resveratrol-induced apoptosis in U937 cells by inhibition of caspase-3 activity. Carcinogenesis 22: 1633-1639, 2001.

25. Inose H, Ochi H, Kimura A, Fujita K, Xu R, Sato S, Iwasaki M, Sunamura S, Takeuchi Y, Fukumoto S, et al: A microRNA regulatory mechanism of osteoblast differentiation. Proc Natl Acad Sci USA 106: 20794-20799, 2009

26. Li XF, Xu H, Zhao YJ, Tang DZ, Xu GH, Holz J, Wang J, Cheng SD, Shi $Q$ and Wang YJ: Icariin augments bone formation and reverses the phenotypes of osteoprotegerin-deficient mice through the activation of Wnt/ $\beta$-catenin-BMP signaling. Evid Based Complement Alternat Med 2013: 652317, 2013.

27. Hsieh TP, Sheu SY, Sun JS and Chen MH: Icariin inhibits osteoclast differentiation and bone resorption by suppression of MAPKs/NF- $\kappa$ B regulated HIF- $1 \alpha$ and PGE(2) synthesis. Phytomedicine 18: 176-185, 2011.

28. Mehl B, Delling G, Schlindwein I, Heilmann P, Voia C, Ziegler R, Nawroth P and Kasperk C: Do markers of bone metabolism reflect the presence of a high- or low-turnover state of bone metabolism? Med Klin 97: 588-594, 2002.

29. Terzi T, Terzi M, Tander B, Cantürk F and Onar M: Changes in bone mineral density and bone metabolism markers in premenopausal women with multiple sclerosis and the relationship to clinical variables. J Clin Neurosci 17: 1260-1264, 2010.

30. Guo Y, Wu X, Zhang X, Li D, Xiao Y, Fan Y and Zhang X: The in vivo study of the effect of icariin on ECM secretion and gene expression of chondrocytes. Pharmacol Clin Chin Mater Med, 2012.

31. Sun P, Liu Y, Deng X, Yu C, Dai N, Yuan X, Chen L, Yu S, Si W, Wang X, et al: An inhibitor of cathepsin K, icariin suppresses cartilage and bone degradation in mice of collagen-induced arthritis. Phytomedicine 20: 975-979, 2013. 
32. Yuan T, He L, Yang J, Zhang L, Xiao Y, Fan Y and Zhang X: Conjugated icariin promotes tissue-engineered cartilage formation in hyaluronic acid/collagen hydrogel. Process Biochem 50: 2242-2250, 2015.

33. Blanco FJ, Geng Y and Lotz M: Differentiation-dependent effects of IL-1 and TGF-beta on human articular chondrocyte proliferation are related to inducible nitric oxide synthase expression. J Immunol 154: 4018-4026, 1995.

34. Roberts AB, Flanders KC, Heine UI, Jakowlew S, Kondaiah P, Kim SJ and Sporn MB: Transforming growth factor-beta: multifunctional regulator of differentiation and development. Philos Trans R Soc Lond B Biol Sci 327: 145-154, 1990.

35. Wang P, Zhang F, He Q, Wang J, Shiu HT, Shu Y, Tsang WP, Liang S, Zhao K and Wan C: Flavonoid compound icariin activates hypoxia inducible factor- $1 \alpha$ in chondrocytes and promotes articular cartilage repair. PLoS One 11: e0148372, 2016.

36. Shantikumar S, Caporali A and Emanueli C: Role of microRNAs in diabetes and its cardiovascular complications. Cardiovasc Res 93: 583-593, 2012.

37. Schoolmeesters A, Eklund T, Leake D, Vermeulen A, Smith Q, Force Aldred $\mathrm{S}$ and Fedorov Y: Functional profiling reveals critical role for miRNA in differentiation of human mesenchymal stem cells. PLoS One 4: e5605, 2009.
38. Bueno MJ, Pérez de Castro I and Malumbres M: Control of cell proliferation pathways by microRNAs. Cell Cycle 7: 3143-3148, 2008.

39. Sumiyoshi K, Kubota S, Ohgawara T, Kawata K, Abd El Kader T, Nishida T, Ikeda N, Shimo T, Yamashiro T and Takigawa M: Novel role of miR-181a in cartilage metabolism. J Cell Biochem 114: 2094-2100, 2013.

40. Mirzamohammadi F, Papaioannou G and Kobayashi T: MicroRNAs in cartilage development, homeostasis, and disease Curr Osteoporos Rep 12: 410-419, 2014.

41. Guérit D, Brondello JM, Chuchana P, Philipot D, Toupet K, Bony $\mathrm{C}$, Jorgensen $\mathrm{C}$ and Noël D: FOXO3A regulation by miRNA-29a controls chondrogenic differentiation of mesenchymal stem cells and cartilage formation. Stem Cells Dev 23: 1195-1205, 2014

42. Zhang L, Zhang X, Li KF, Li DX, Xiao YM, Fan YJ and Zhang XD: Icariin promotes extracellular matrix synthesis and gene expression of chondrocytes in vitro. Phytother Res 26: 1385-1392, 2012. 